\title{
SFRP1 inhibited the epithelial ovarian cancer through inhibiting Wnt/B-catenin signaling
}

\author{
Hao Zhang ${ }^{1 *}$, Duohe Sun ${ }^{* *}$, Jianping Qiu3 ${ }^{\square}$ and Liangqing Yao ${ }^{\square}$ \\ 1Department of Gynecology, Obstetrics and Gynecology Hospital, Fudan University, Shanghai, 200011, P.R. China; ${ }^{2}$ Department of Obstetrics \\ and Gynecology, Shanghai Jiaotong University, Affiliated Sixth People's Hospital South Campus, Shanghai, 201499, P.R. China; ${ }^{3}$ Department of \\ Obstetrics and Gynecology, Nanjing Medical University Hospital Affiliated Suzhou, Suzhou 215008, P.R. China
}

\begin{abstract}
Background: Epithelial ovarian cancer is the most malignant gynecologic neoplasm accounting for $90 \%$ of the ovarian cancer patients. Objective: Researchers proved that epigenetic alterations could disrupt gene expression as often as genetic alterations. Secreted frizzed related protein (SFRP1), a Wnt antagonist, exerts a significant effect on ovarian cancer. The aim of this research was to investigate the effects and the mechanism of action of SFRP1 on epithelial ovarian cancer. Methods: Clinical specimens (including fallopian tubes epithelium from 60 epithelial ovarian cancer patients' and 20 healthy subjects who were undergoing surgical treatments), transgenic mice (overexpressing SFRP1 gene), and 4 epithelial ovarian cancer cell lines (including OVCAR4, SKOV3, COV644, TOV21G) were used in this study. Overexpression of SFRP1 in cells was carried out on OVCAR4 cells by transfection using Lipofectamine 2000. Gene transcription was analyzed by qRT-PCR. The methylation of SFRP1 gene was quantified by methylation-specific PCR. The level of protein expression was measured by Western blot or immunohistochemistry analysis. Cell proliferation was analyzed by CCK8 methods. The ability of cell migration and invasion were measured by wound healing assay and transwell assay. Results: Abnormal expression level and hypermethylation status of SFRP1 were found in clinical epithelial ovarian cancer samples and cell lines. We observed that SFRP1 knockdown could promote proliferation, migration and invasion abilities of epithelial ovarian cancer cells. Additionally, we discovered a potential inhibitory effect of SFRP1 on Wnt/ $\beta$-catenin signaling pathway in epithelial ovarian cancer cells. Furthermore, the anti-tumor effect of SFRP1 was tested in SFRP1 transgenic mice. Conclusion: SFRP1 inhibited epithelial ovarian cancer through inhibiting Wnt/ $\beta$-catenin pathway, suggesting that SFRP1 could be considered as a potential therapeutic target in epithelial ovarian cancer.
\end{abstract}

Key words: SFRP1, methylation, Wnt/ $\beta$-catenin, epithelial ovarian cancer

Received: 20 December, 2018; revised: 21 June, 2019; accepted: 08 October, 2019; available on-line: 26 November, 2019

『e-mail: JianpingQiuzxc@163.com (JQ); LiangqingYaozxc@163.com (LY)

${ }^{*}$ Co-first authors

Abbreviations: DKK, Dickkopf-related protein; DMEM, dulbecco's modified eagle medium; IHC, immunohistochemistry analysis; MSP, methylation-specific PCR (MSP); PARP, poly ADP-ribose polymerase inhibitors; qRT-PCR, quantitative reverse transcription-polymerase chain reaction; SFRP1, secreted frizzed related protein

\section{INTRODUCTION}

Originating in the endometrium, epithelial ovarian cancer is thought to arise from the fallopian tube rather than from ovary or other pelvic organs (Cho \& Shih, 2009; Lheureux et al., 2019). Similarly to other types of epithelial neoplasms, epithelial ovarian cancer could metastasize by lymphatics, renal hilus or peritoneum (Lheureux et al., 2019). Epithelial ovarian cancer is proposed as the most malignant gynecologic neoplasm, accounting for $90 \%$ ovarian cancer patients (Kurman \& Shih, 2010). To date, the mainstream therapeutic method is chemotherapy, including single non-platinum-based agents, taxanes, and poly ADP-ribose polymerase inhibitors (PARP), surgery, including oophorectomy (Baylin \& Herman, 2000). However, given many factors, including endocrine disorders and immune dysfunction, epithelial ovarian cancer patients often suffer from tumor recurrence (Morgan et al., 2011). The molecular pathogenic mechanisms underlying epithelial ovarian cancers are complicated, including the gene mutations, endocrine etiology, etc. Interestingly, abnormalities of gene function or chromosome segments are considered as the most important risk factors (Kurman \& Shih, 2011). Gene function in the context of cancer might be disrupted by epigenetic modifications or by genetic alterations (Baylin et al., 2001), as the classic genetics theory alone cannot explain the diversity of cancer subtypes. It is well established that aberrant patterns of gene expression, epigenetic modification of DNA and chromatin conformation play an important role in the tumor. Among which, the DNA hypermethylation and hypomethylation are the research hotspots (Baylin et al., 2001).

An extracellular signaling molecule named secreted frizzed related protein (SFRP1), was reported to be a modulator of the important cell signaling pathway in which the Wnt ligand bounds to frizzled membrane receptors (Katoh \& Katoh, 2006). Hypermethylation of SFRP gene was observed in ovarian cancer, colon cancer, acute myeloid leukemia (Ba et al., 2017). Interestingly, SFRP is a Wnt antagonist that modulates Wnt signaling pathway (Holly et al., 2014). Furthermore, the effects of SFRP on Wnt signaling pathway were reported under physiological or pathological conditions. Both bone mass and metabolism and cancer and systemic sclerosis could be regulated by SFRP/Wnt signaling (Dees et al., 2014). Additionally, Wnt signaling participates in the development of epithelial neoplasms through increasing the expression of proliferation-associated proteins, such as c-Myc and cyclin D1 (Cowling et al., 2007). However, the influence of epigenetic inactivation of SFRP gene on Wnt signaling pathway and effects on epithelial ovarian 
cancer remain unknown. In this study, with the aim to clarify the effects of epigenetic alterations of SRFP on ovarian cancer, we reported abnormal expression levels and methylation status of SFRP1 in patients. We further explored the effect of SFRP1 overexpression or knockdown in cell models. Moreover, the regulation of SFRP1 was mediated by Wnt/ $\beta$-catenin signaling pathway. Interestingly, SFRP1 gene overexpression could inhibit the growth of epithelial ovarian cancer in vivo.

\section{MATERIALS AND METHODS}

Experimental subjects. Clinical specimens. A total of 60 epithelial ovarian cancer patients' fallopian tubes epithelium and 20 healthy subjects' fallopian tubes epithelium were obtained from the Chinese female subjects (Peking University People's Hospital, Beijing, China) who were undergoing surgical treatments. All the specimens were ethically justified. All fallopian tube epithelial cell samples were conserved in physiological saline solution after brushing and frozen immediately.

Reagents. The inhibitor of $\beta$-catenin named XAV939 was purchased from Selleck Chemical (S1180).

Cell lines. Four epithelial ovarian cancer cell lines were used in this study, named OVCAR4, SKOV3, COV644, TOV21G. OVCAR4 and SKOV3 were purchased from R\&D Systems (Minneapolis, MN). COV644 and TOV21G were bought from American Type Culture Collection (ATCC, Manassas, VA). Cells were maintained at $37^{\circ} \mathrm{C}$ with $5 \% \mathrm{CO}_{2}$, and cultured in Roswell Park Memorial Institute-1640 medium (RPMI-1640) (Gibco, Grand Island, NY) supplemented with 10\% Fetal Bovine Serum (FBS) (Gibco, Grand Island, NY), 100 IU penicillin and streptomycin. Human ovarian surface epithelial (HOSE) was chosen as the control cells. HOSE cells were established by immortalizing normal human ovarian surface cells with papilloma virus E6/E7, and cultured in DMEM supplemented with $10 \%$ FBS and $50 \mu \mathrm{g} / \mathrm{mL}$ gentamicin sulfate.

Animals. Transgenic mice overexpressing SFRP1 gene were purchased from Cyagen Biosciences (Guangzhou, China) and were crossed more than 10 generations. The implantation experiment was performed in the 8-weekold transgenic mice. All the experimental procedures were approved by the Obstetrics and Gynecology Hospital Ethics Committee and the institutional animal care and use committee. The animal experiments were in full compliance with the European Communities Council Directive of 24 November 1986 (86/609/EEC) or with the Guidelines laid down by the NIH in the US.

Establishment of epithelial ovarian cancer animal model. 8-week-old nude mice (Charles River Laboratories, Beijing, China) were utilized to generate the ovarian cancer model. Mice were irradiated with neutron source using a dose of 2.7 Gy. After 1-2 month, ovarian and subcutaneous tumor cells were extracted from the irradiated mice and then implanted into the SFRP1 transgenic mice and wild-type mice. The size and volume of the tumor were assessed 1 month post implantation.

SFRP1 plasmid construction and transfection. The pCMV6 and pCMV6-SFRP1 plasmids were bought from ORIGENE (Rockville, MD). SFRP1 overexpression was carried out on OVCAR4 cells by transfecting with empty pCMV6 vector as the negative control (NC) and pCMV6-SFRP1 using Lipofectamine 2000 (ThermoFisher, Cheshire, UK) as in the previous work (Kim et al., 2016). Cells were selected in medium supplemented with
Geneticin G418 (ThermoFisher Cheshire, UK) for six weeks.

Knockdown of SFRP1 was conducted on OVCAR4 cells by RNAi transfection. The oligonucleotide fragments of the negative control (siNC) and siRNA targeting SFRP1 (siSFRP1) were purchased from GENECHEM (Shanghai, China). OVCAR4 cells were transiently transfected with siSFRP1 using FuGENE HD (Roche, Shanghai, China). Medium was replaced with fresh DMEM 8 hours post transfection.

Isolation of genomic DNA. The genomic DNA was isolated from fallopian tubes epithelium of epithelial ovarian cancer patients and healthy subjects as well as from the epithelial ovarian cancer cell lines. The procedure was performed using DNeasy Blood and Tissue kit (Qiagen, Düsseldorf, Germany) according to the manufacturer's instructions. The isolated genomic DNA was processed for methylation-specific PCR.

Methylation-specific PCR (MSP). The genomic DNA was treated with bisulfite for the bisulfite modification of DNA. EpiTect Fast DNA Bisulfite Kit (Qiagen, Düsseldorf, Germany) was used to convert cytosine to uracil in the unmethylated genomic DNA. We used MethPrimers software to design 5'-TGTAGTTTTCGGAGT'TAGTGTCGCGC-3' and 5'-CCTACGATCGAAAACGACGCGAACG-3' (amplicon length: 126 bp; annealing temperature: $60^{\circ} \mathrm{C}$ ) primers for the methylated sequence of SFRP1, and 5'-GTT'T'TGTAGTTT'T'TGGAGTTAGTGTTGTGT-3' and 5'-CTCAACCTACAATCAAAAACAACACAAACA-3' (amplicon length $135 \mathrm{bp}$; annealing temperature: $60^{\circ} \mathrm{C}$ ) primers for the unmethylated sequence of SFRP1.

qRT-PCR (quantitative reverse transcriptionpolymerase chain reaction). Total RNA from HOSE, OVCAR4, SKOV3, COV644, TOV21G cells and fallopian tubes epithelium was extracted using Trizol Reagent (Invitrogen, Cheshire, UK). RNA was reverse-transcribed into cDNA using Super Script reverse transcriptase (ThermoFisher, Cheshire, UK). To assess the mRNA level and the transfection efficiency, qRT-PCR analysis was performed with the following primers: SFRP1 forward, 5'-GATGCT'TAAGTGTGACAAGT'TCC-3' and SFRP1 reverse, 5'-TCAGAT'T'TCAACTCGT'TGTCACAG-3' (amplicon length: 130 bp; annealing temperature: $62^{\circ} \mathrm{C}$ ); glyceraldehyde-3-phosphate dehydrogenase $(G A P D H)$ forward, 5'-AGGTGAAGGTCGGAGTCAACG -3' and GAPDH reverse, 5'-AGGGGTCATTGATGGCAACA-3' (amplicon length: $130 \mathrm{bp}$; annealing temperature: $62^{\circ} \mathrm{C}$ ). The transfection efficiency of SFRP1 siRNA was $\sim 70 \%$.

Western blot. Proteins were extracted from OVCAR4 cells post-transfection using lysis solution containing Tris- $\mathrm{HCl}$ (20 mM, pH=7.5), Triton X-100 (1\%), PMSF (1\%). After total protein estimation BCA Protein Assay Kit (ThermoFisher, Cheshire, UK), protein samples were separated by $10 \%$ SDS-PAGE, then transferred onto the PVDF membranes. Membranes were incubated with rabbit anti-SFRP1 antibody (Abcam, Cambridge, MA) and goat anti-c-Myc antibody, goat anti-Cyclin D1 antibody, goat anti- $\beta$-catenin antibody, goat anti-GAPDH antibody (Abcam, Cambridge, MA) for $16 \mathrm{~h}$ at $4^{\circ} \mathrm{C}$, and subsequently with secondary anti-goat HRP-conjugated antibody for $1 \mathrm{~h}$ at $22^{\circ} \mathrm{C}$.

Immunohistochemistry analysis (IHC). Immunohistochemistry analysis was used to test the cell proliferation and invasion and SFRP1 protein expression in the epithelial ovarian cancer tissue. The anti-SFRP1 antibody for IHC was bought from Millipore (Bedford, MA). Pa- 

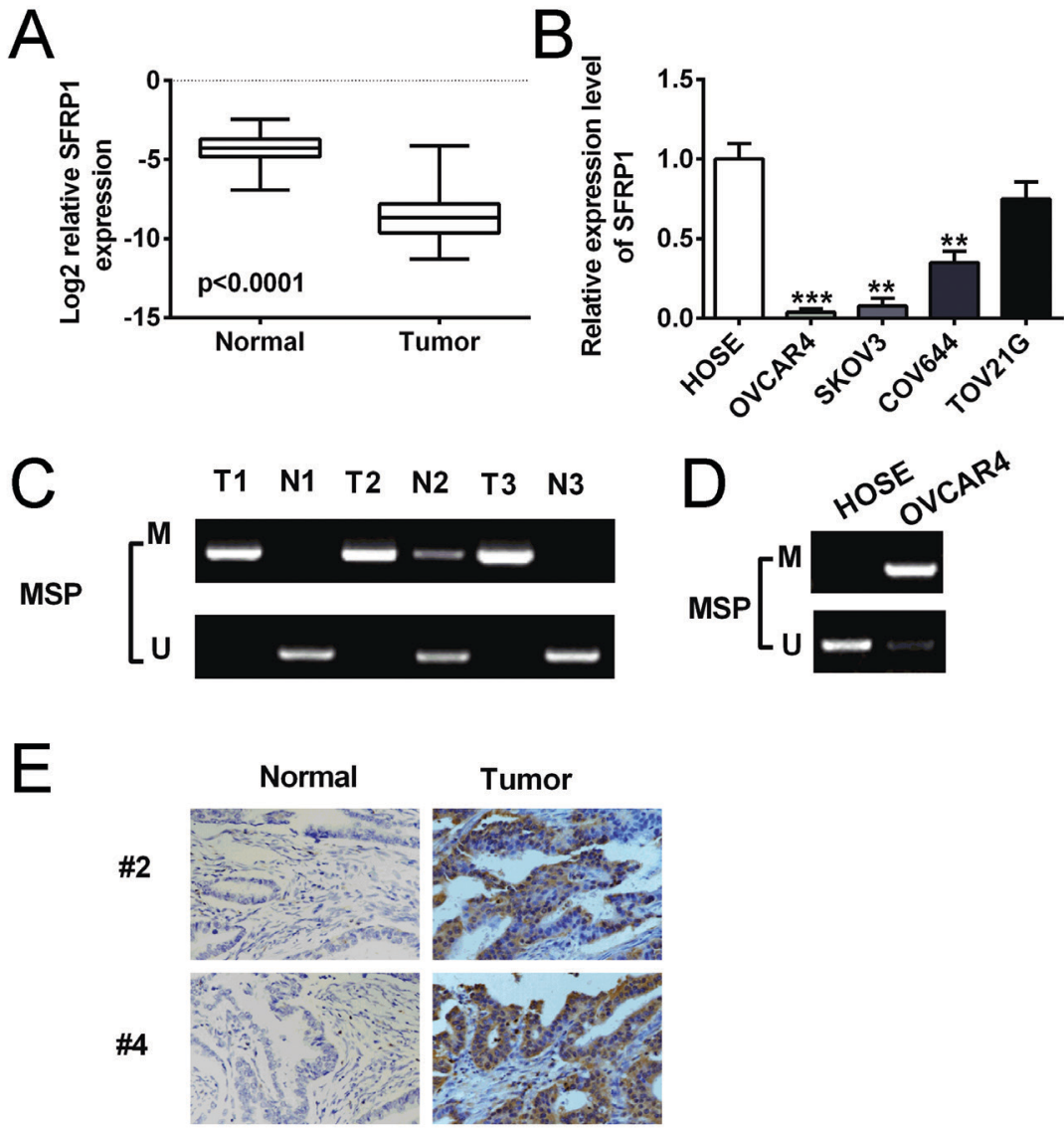

Figure 1. Aberrant expression level and methylation status of SFRP1.

(A) $\mathrm{Log}_{2}$ relative mRNA level of SFRP1. (B) Relative SFRP1 mRNA levels in five cell lines. (C) DNA methylation status of SFRP1 in clinical samples. (D) DNA methylation status of SFRP1 in OVCAR4 cells. (E) IHC analysis showing SFRP1 protein expression in the fallopian tube. $\left(\mathrm{n}=10\right.$; One-way ANOVA analysis; $\left.{ }^{*} p<0.05,{ }^{* *} p<0.01,{ }^{* * *} p<0.001\right)$.

tients' tissues were embedded in paraffin after the conventional treatment, then subjected to IHC analysis.

Post transfection, OVCAR4 cells were seeded onto a 6-well plate. Cells were incubated with DAPI (Abcam, Cambridge, MA) for $30 \mathrm{~min}$. After the Transwell assay, the membranes were fetched and incubated with DAPI for $30 \mathrm{~min}$.

Cellular proliferation assay. $1.5 \times 10^{6}$ OVCAR 4 cells were seeded onto 12 -well plates in DMEM medium. $36 \mathrm{~h}$ post-seeding cells were washed with $1 \times \mathrm{PBS}$, and incubated with DAPI for $30 \mathrm{~min}$, observed and counted under a microscope.

The CCK-8 assay was also conducted to test cell proliferation. $1 \times 10^{5}$ cells were plated in 96-well plates and incubated with cell counting kit- 8 solutions (R\&D Systems, Minneapolis, MN) for $1.5 \mathrm{~h}$ at $37^{\circ} \mathrm{C}$.

Migration and invasion assay. Wound healing assay was performed to evaluate the cell migration of OVCAR4 cells upon SFRP1 overexpression or knockdown. Briefly, $2 \times 10^{6}$ OVCAR4 cells were plated in a 6-well plate with RPMI-1640 medium. When the cell confluence reached $\sim 80 \%$, a line in the middle plates was scratched using sterile pipette tip, and washed three times. IncuCyte system (ESSEN, American) was used to observe the wound width.

Transwell assay was conducted to study the cell invasion of OVCAR4 cells upon SFRP1 overexpression or knockdown. Transwell chambers were inserted into the 6 -well plate. The pore diameter of the membrane was 3.0 $\mu \mathrm{m}$, and the membrane was coated with Matrigel. $36 \mathrm{~h}$ post-seeding, membranes were incubated with DAPI for $30 \mathrm{~min}$, observed and quantified under a microscope.

Statistical analysis. All the values in this paper were presented as mean \pm standard deviation (S.D.), and analyzed by SPSS 18.0. One-way ANOVA and Dunnett's post hoc test were used to compare several groups to the control. $p<0.05$ was considered statistically significant in this study $(* p<0.05, * * p<0.01, * * * p<0.005$, **** $p<0.001)$.

\section{RESULTS}

\section{Expression level and methylation status of SFRP1 in epithelial ovarian cancer patients and cell lines}

Clinical fallopian tubes epithelium samples and the four epithelial ovarian cancer cell lines were collected or purchased as described above. Total RNA was extracted for qRT-PCR analysis. The methylation of SFRP1 promoter was analyzed in 4 ovarian cancer cell lines and in fallopian tubes epithelium of epithelial ovarian cancer patients by MSP. Results showed that SFRP1 mRNA level in fallopian tube epithelium of epithelial ovarian cancer patients was lower than in healthy subjects $(* * * * p<0.0001)$ (Fig. 1A). We also tested the SFRP1 mRNA levels in four epithelial ovarian cancer cell lines (OVCAR4, SKOV3, COV644, TOV21G) and a control cell line named HOSE, and we observed lower SFRP1 mRNA levels in the four epithelial ovarian cancer cell lines in comparison to HOSE. It is worth mentioning that SFRP1 mRNA level in OVCAR4 

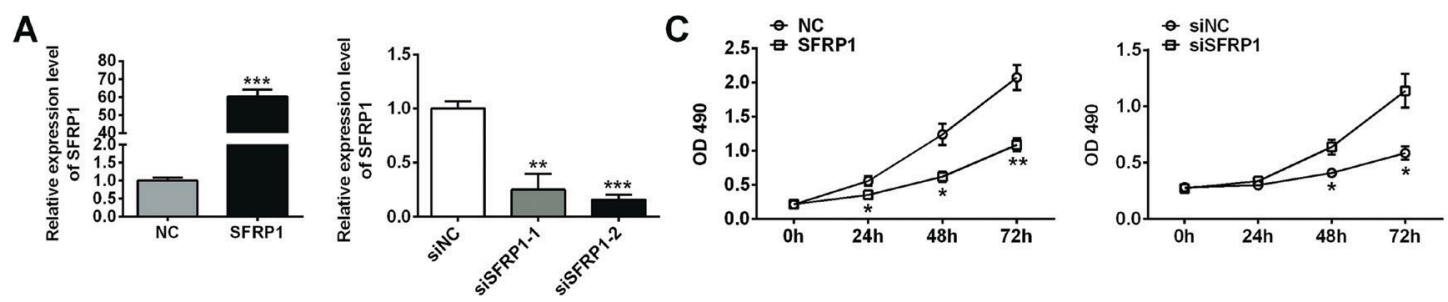

B
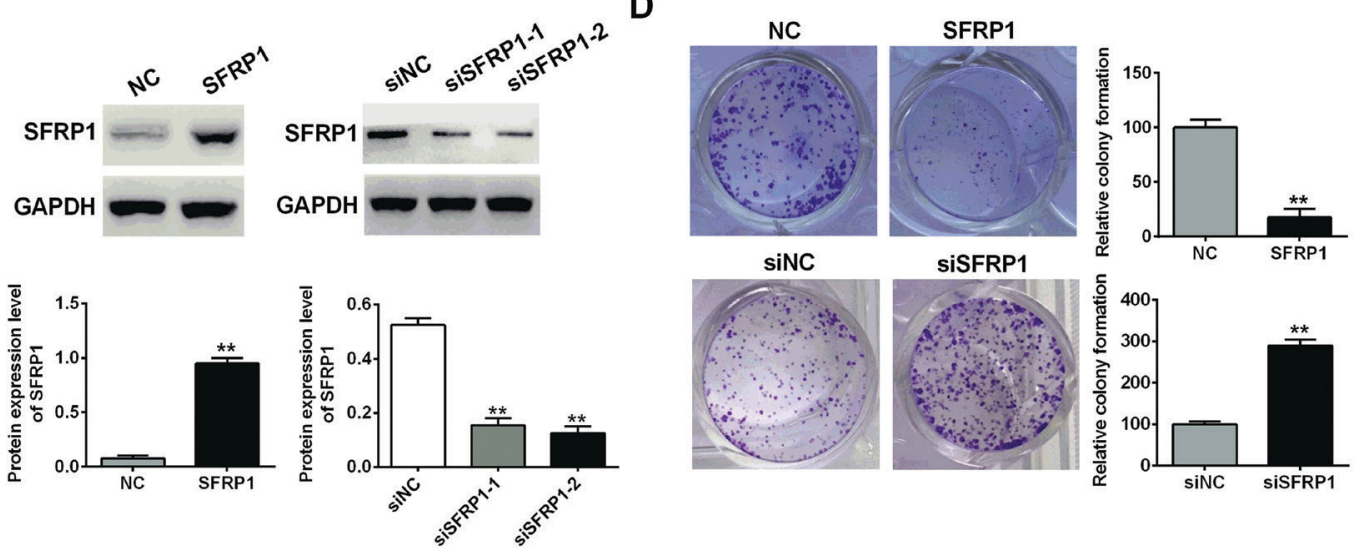

siSFRP1

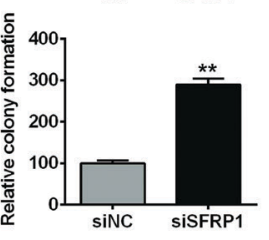

Figure 2. Effects of SFRP1 on cells proliferation.

(A) SFRP1 mRNA level upon SFRP1 overexpression and knockdown. (B) SFRP1 protein level upon SFRP1 overexpression and knockdown. (C) OVCAR4 cells activity upon SFRP1 overexpression and knockdown. (D) OVCAR4 cells number upon SFRP1 overexpression and knockdown. ( $\mathrm{n}=7$; One-way ANOVA analysis; $\left.{ }^{*} p<0.05,{ }^{* *} p<0.01,{ }^{* * *} p<0.001\right)$.

A
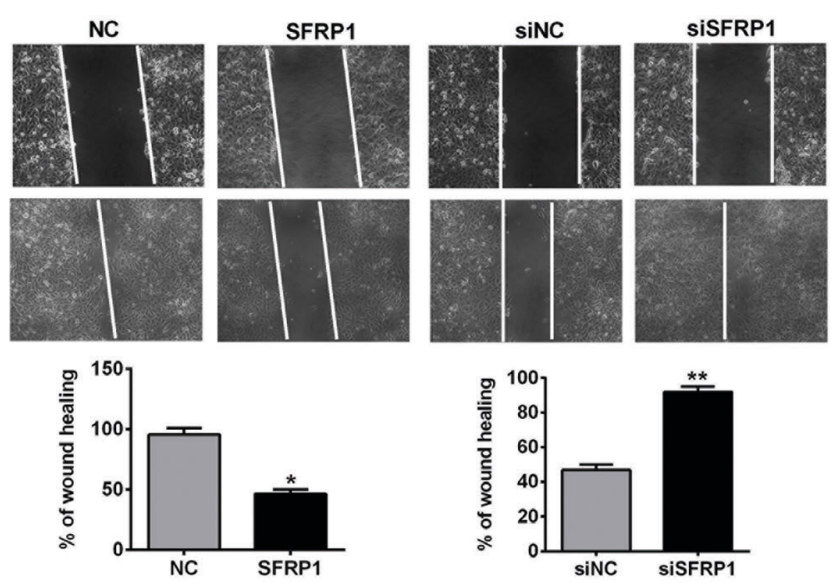

B

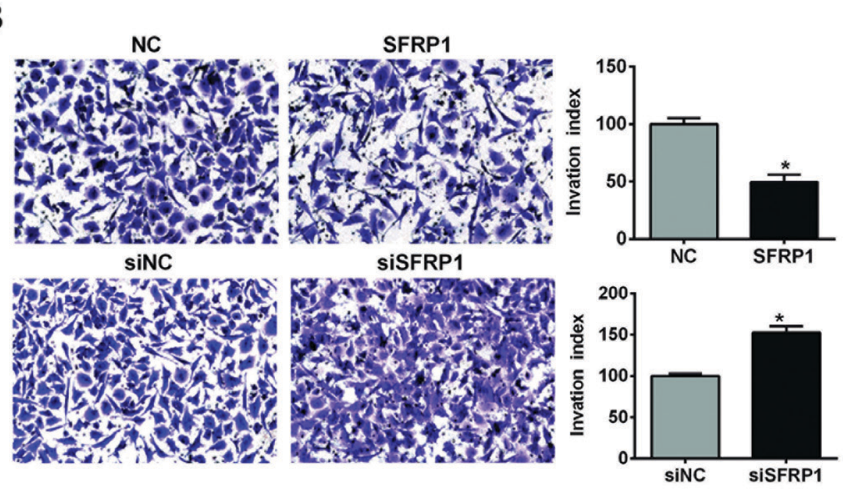

Figure 3. Effects of SFRP1 on cell migration and invasion.

(A) The migration rate of OVCAR4 cells upon SFRP1 overexpression and knockdown. (B) Invasion ability of OVCAR4 cells upon SFRP1 overexpression and knockdown. ( $\mathrm{n}=7$; One-way ANOVA analysis; $\left.{ }^{*} p<0.05,{ }^{* *} p<0.01,{ }^{* * *} p<0.001\right)$. 
A

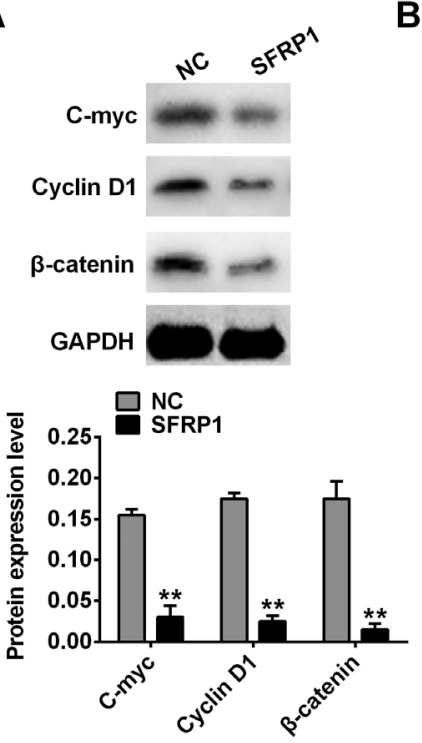

B
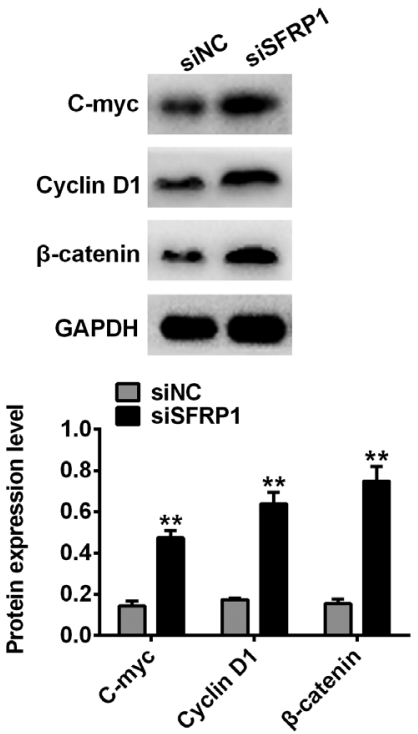

Figure 4. SFRP1 modulated Wnt signaling pathway in epithelial ovarian cancer cells.

(A) Effect of SFRP1 overexpression on Wnt signaling pathway proteins: $\beta$-catenin, C-myc, Cyclin D1. (B) Effect of SFRP1 knockdown on Wnt signaling pathway proteins: $\beta$-catenin, C-myc, Cyclin D1. ( $\mathrm{n}=7$; One-way ANOVA analysis; ${ }^{*} p<0.05,{ }^{* *} p<0.01,{ }^{* * *} p<0.001$ ).

A
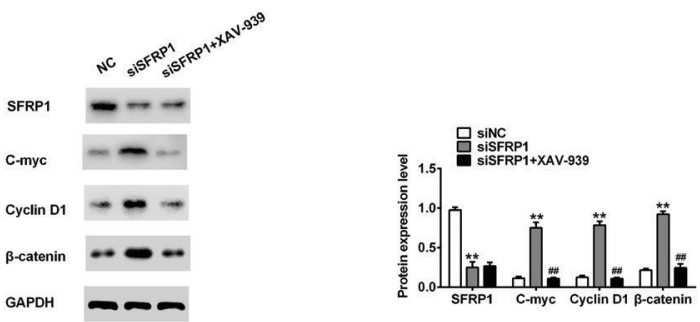

B

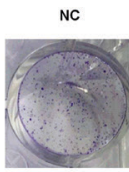

siSFRP1
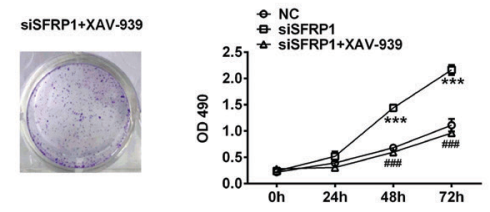

C
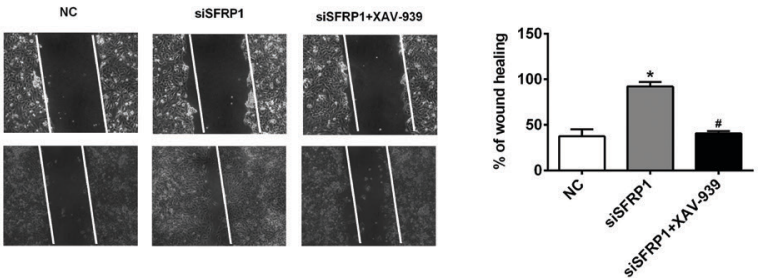

D
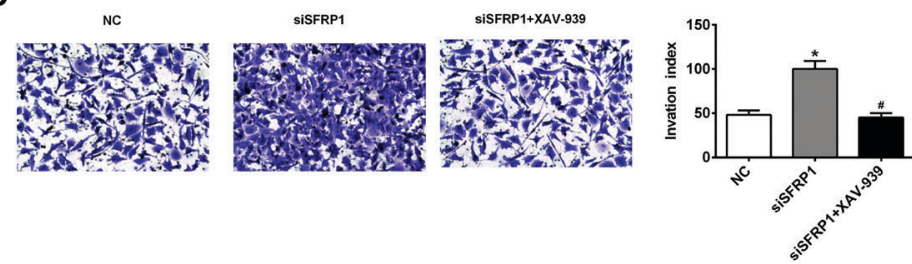

Figure 5. SFRP1 influenced cells proliferation, migration and invasion through modulating Wnt signaling pathway.

(A) Effect of $\beta$-catenin inhibitor, XAV-939 and SFRP1 knockdown on Wnt signaling pathway proteins: $\beta$-catenin, C-myc, Cyclin D1. (B) Effect of $\beta$-catenin inhibitor, XAV-939 and SFRP1 knockdown on the proliferation of OVCAR4 cells. (C) Effect of $\beta$-catenin inhibitor, XAV-939 and SFRP1 knockdown on the migration of OVCAR4 cells. (D) Effect of $\beta$-catenin inhibitor, XAV-939 and SFRP1 knockdown on the invasion of OVCAR4 cells. ( $n=7$; One-way ANOVA analysis; ${ }^{*} p<0.05,{ }^{* *} p<0.01,{ }^{* * *} p<0.001$ ). 
A

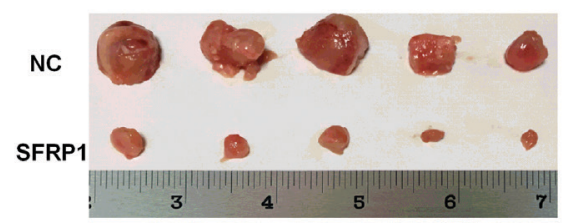

B

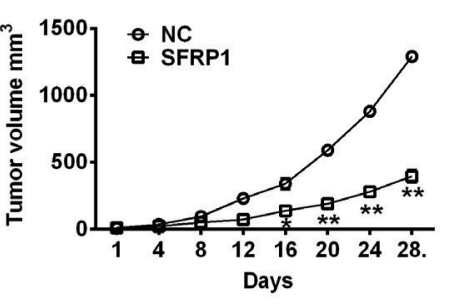

C
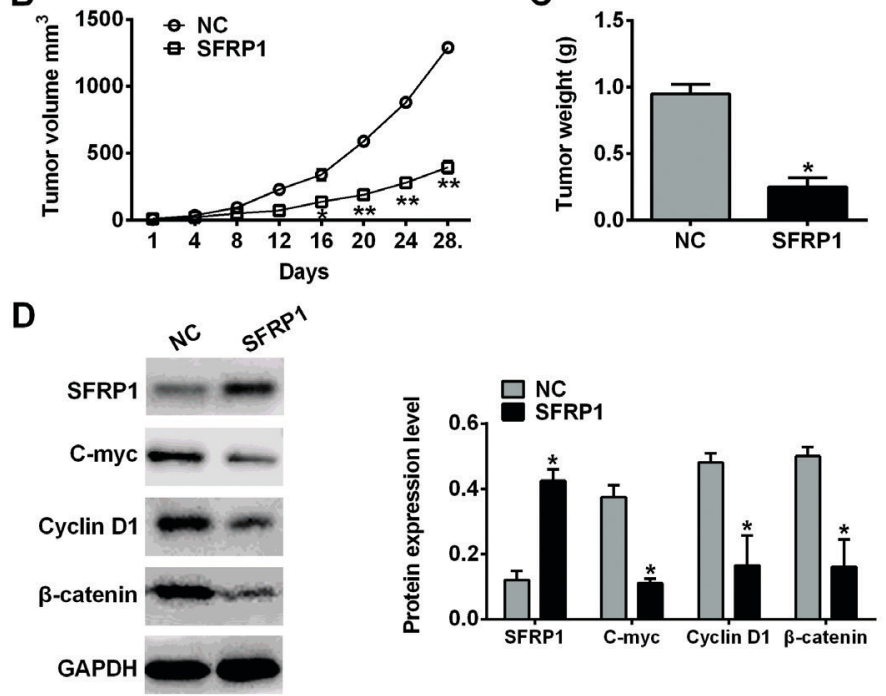

Figure 6. SFRP1 suppressed tumor growth and the expression of key proteins involved in Wnt signaling.

(A) Effect of SFRP1 overexpression on tumor size. (B) Effect of SFRP1 overexpression on tumor volume. (C) Effect of SFRP1 overexpression on tumor weight. (D) Effect of SFRP1 overexpression on the level of Wnt signaling pathway proteins: $\beta$-catenin, C-myc, Cyclin D1 in the tumor. ( $\mathrm{n}=10$; One-way ANOVA analysis; ${ }^{*} p<0.05,{ }^{* *} p<0.01,{ }^{* * *} p<0.001$ ).

cells was the lowest (Fig. 1B). Therefore, we chose OVCAR4 cells as our model cells.

Extensive research reported the aberration of SFRP1 gene expression in cancer due to abnormal methylation status (Sugiyama et al., 2013). To further investigate the relationship between SFRP1 mRNA level and methylation, we performed MSP analysis to test the methylation level in clinical fallopian tubes epithelium samples and OVCAR4 cells. Results showed that methylation levels of SFRP1 gene in fallopian tubes epithelium of epithelial ovarian cancer patients and OVCAR4 cells were higher than in negative control (Fig. 1C-D). Histological staining indicated that SFRP1 protein was decreased in the fallopian tubes epithelium of epithelial ovarian cancer patients (Fig. 1E). Collectively, our data suggest that low expression level of SFRP1 is elevated in epithelial ovarian cancer due to the abnormal methylation of the SFRP1 gene.

\section{Effects of SFRP1 on cells proliferation}

To further investigate the effects of SFRP1 on epithelial ovarian cancer, we performed overexpression and knockdown of SFRP1 in OVCAR4 cells. We tested the SFRP1 mRNA and protein level in the OVCAR4 cells, showing that transfection using pCMV6-FoxQ1 plasmid successfully changed SFRP1 mRNA level and SFRP1 protein level (Fig. 2A, B). Moreover, CCK-8 assay was performed to test the cell viability and proliferation. Consistently, SFRP1 overexpression inhibited, while SFRP1 knockdown promoted the viability and proliferation of OVCAR4 cells (Fig. 2C, D).

\section{Effects of SFRP1 on cell migration and invasion}

To further explore the influence of SFRP1 on the migration and invasion, we performed wound healing assay and trans-well assay. Wound healing assay results suggested that SFRP1 overexpression significantly inhibited the migration ability of OVCAR4 cells, while SFRP1 knockdown significantly promoted the migration ability (Fig. 3A). Transwell assay demonstrated that SFRP1 overexpression significantly decreased the invasion rate of OVCAR4 cells, while SFRP1 knockdown significantly elevated the invasion rate (Fig. 3B).

\section{Effects of SFRP1 on Wnt signaling}

According to the previous studies, SFRP1 is considered an antagonist of Wnt signaling, and the aberrant methylation of SFRP1 gene caused hyperactivation of Wnt pathway (Yamaguchi et al., 2015). In our experiments, we investigated the effect of SFRP1 on Wnt signaling pathway protein $\beta$-catenin and proliferationassociated proteins c-Myc and cyclin D1 by western blot. Results showed that SFRP1 overexpression decreased $\beta$-catenin level, which was accompanied by the reduced expression of proliferation-associated proteins c-Myc and cyclin D1 (Fig. 4A). However, SFRP1 knockdown elevated $\beta$-catenin protein level, which was accompanied by the increased expression of c-Myc and cyclin D1 (Fig. 4B).

To further confirm the effect of SFRP1 on Wnt signaling, we next investigated Wnt signaling pathway-related proteins level upon SFRP1 knockdown. According to the previous results, the proliferation, migration and invasion ability of OVCAR4 cells were increased upon SFRP1 knockdown. We used the inhibitor of $\beta$-catenin, XAV-939 to study the effect of SFRP1 knockdown without $\beta$-catenin activity. Interestingly, results indicated that SFRP1 knockdown promoted the proliferation, migration and invasion of OVCAR4 cells (Fig. 5B, C, D), 
but XAV-939 could reverse such SFRP1-mediated cellular events, which was followed by the decreased c-Myc and cyclin D1 protein expression (Fig. 5A).

\section{Effects of SFRP1 on tumor growth}

Given the inhibitory effect of SFRP1 on epithelial ovarian cancer cells, we hypothesized that SFRP1 could also inhibit the tumor growth in vivo. With the aim to study the effect of SFRP1 in vivo, we subcutaneously injected the epithelial ovarian cancer cells to establish an epithelial ovarian cancer model in SFRP1 transgenic mice and wild mice. Intriguingly, the size, volume and weight of tumor were decreased in SFRP1 transgenic mice (Fig. 6A, B, C). Moreover, we further discovered that $\beta$-catenin protein level was also lowered in SFRP1 transgenic mice, which was accompanied by the reduced c-Myc and cyclin D1 levels (Fig. 6D).

\section{DISCUSSION}

In this study, we unraveled a link between SFRP1, Wnt signaling and epithelial ovarian cancer. We observed aberrant expression level and methylation status of SFRP1 in both epithelial ovarian cancer tissues cell models, which were consistent with previous studies. The aberrant expression and methylation of SFRP1 in cancer were reported extensively: Vedran Kardum et al. reported the loss of SFRP1 protein expression observed in low-grade serous ovarian carcinomas (Kardum et al., 2017). The CpG island hypermethylation of SFRP1 is a common event observed in many kinds of cancers (Atschekzei et al., 2012), which might be one of the key reasons for the lower expression in epithelial ovarian cancer. Jasenka Partl and others (Partl et al., 2014) reported that loss of SFRP1 and SFRP3 expression was involved in the development of human trophoblastic tumors. We analyzed the clinical epithelial ovarian cancer tissue samples and cell models and confirmed the lower expression of SFRP1 in epithelial ovarian tumor. Furthermore, we verified the relationship between SFRP1 and epithelial ovarian cancer and proved that SFRP1 protein could inhibit epithelial ovarian cells in vitro.

The effect was observed in other cancers such as colon cancer and pancreatic cancer (Bu et al., 2008a, Wang et al., 2018), SFRP1 was considered as a factor inhibiting the tumor growth through Wnt signaling modulation. Moreover, epigenetic inactivation of SFRP1 via hypermethylation could be a shared event. Other anti-tumor factors like Dickkopf-related protein 1 (DKK1) could also be methylated in $\mathrm{CpG}$ sequences of the promoter (Revet et al., 2010). Briefly, epigenetics alterations should not be neglected in cancer, especially the DNA methylation (Esteller, 2008). DNA methylation is the best known epigenetic marker, which plays an indispensable role in genetic expression. For example, the certain tissue-specific genes: MASPIN (a member of the serum protease inhibitor family) and $M A G E$ (germ-line genes) are silent in almost all tissues (Berardi et al., 2013). However, hypomethylation and expression of $M A S P I N$ and $M A G E$ genes were detected in cancer cells (Tellez et al., 2009). The hypomethylation of DNA was one of the first epigenetic alterations to be found in human cancer, whose the major reason is $\mathrm{CpG}$ islands hypermethylation of tumor-suppressor genes (Tellez et al., 2009). In addition, histone modifications such as acetylation and methylation play an essential role in cancer (Esteller, 2007). Hence, we hypothesized that epigenetic regulations might promote cancer. Nevertheless, the DNA methylation degree varies with each individual and cell line. In our paper, we chose six subjects to perform the MSP analysis, and the results showed that N2 (Fig. 1C) was different from the others. Additionally, cell lines displayed diversity in DNA methylation status and protein level, as OVCAR4 cells were characterized by the highest DNA methylation degree and lowest SFRP1 mRNA level. However, it was not clear whether the cause of the difference was an individual variation or the status of the cells. Considerably, the cell cycle might be controlled in the phase of pre-mRNA or protein synthesis. And the different grades of tumor might be the reason for the different DNA methylation degree, similarly as glutathione S-transferase gene which has different methylation status at the early and late stages of prostate cancer (Lee et al., 1994, Jeronimo et al., 2001).

We also discovered that SFRP1 inhibited tumor growth through regulating Wnt signaling, including $\beta$-catenin, cyclin D1, and C-myc. SFRP1 could competitively bind to $\beta$-catenin receptor, $\mathrm{Fz}$ or directly influence the Wnt-protein functions (Satoh et al., 2010). Cyclin D1 is a nuclear protein required for cell cycle progression in G1 phase, while the anti-cyclin D1 antibody could prevent cells from entering $\mathrm{S}$ phase. Cyclin D1 gene is a target of $\beta$-catenin (Tetsu \& Mccormick, 1999). C-myc, another downstream target of $\beta$-catenin, inhibits epithelialization and wound healing (Stojadinovic et al., 2005). Hence, inhibition of the $\beta$-catenin expression by SFRP1 was accompanied by the decreased cyclin D1 and C-myc levels, which might partially explain SFRP1-mediated anti-tumor effects. Similarly to SFRP1, other SFRPs family and DKKs family members also function as Wnt antagonists in different tissues and in the context of various diseases (Revet et al., 2010). SFRP1 gene overexpression could prominently improve the epithelial ovarian cancer in the animal model, indicating that SFRP1 could be a key therapeutic target in epithelial ovarian cancer.

\section{CONCLUSION}

We demonstrated an aberrant expression level and methylation status of SFRP1 in both epithelial ovarian cancer tissues and cell models. We reported that SFRP1 influenced the proliferation, migration and invasion via inhibiting $\beta$-catenin signaling, which was accompanied by the decreased expression levels of cyclin D1 and Cmyc. Importantly, SFRP1 could inhibit epithelial ovarian cancer growth in vivo. Collectively, this study shed light on SFRP1 as a potential therapeutic target of epithelial ovarian cancer treatment from bench to clinic.

\section{Conflict of interest}

The authors declare no conflict of interests.

\section{REFERENCES}

Atschekzei F, Hennenlotter J, Jänisch S, Großhennig A, Tränkenschuh W, Waalkes S, Peters I, Dörk T, Merseburger AS, Stenzl A, Kuczyk MA, Serth J (2012) SFRP1 CpG island methylation locus is associated with renal cell cancer susceptibility and disease recurrence. Epigenetics 7: 447-457. https://doi.org/10.4161/epi.19614

Ba S, Xuan Y, Long ZW, Chen HY, Zheng SS (2017) MicroRNA-27a promotes the proliferation and invasiveness of colon cancer cells by targeting SFRP1 through the Wnt/ $\beta$-Catenin signaling pathway. Cell Physiol Biochem 42: 1920-1933. https://doi.org/10.1159/000479610

Baylin SB, Herman JG (2000) DNA hypermethylation in tumorigenesis: epigenetics joins genetics. Trends Genet 16: 168-174. https://doi. org/10.1016/s0168-9525(99)01971-x

Baylin SB, Esteller M, Rountree MR, Bachman KE, Schuebel K, Herman JG (2001) Aberrant patterns of DNA methylation, chromatin 
formation and gene expression in cancer. Hum Mol Genet 10: 687692. https://doi.org/10.1093/hmg/10.7.687

Berardi R, Morgese F, Onofri A, Mazzanti P, Pistelli M, Ballatore Z, Savini A, De Lisa M, Caramanti M, Rinaldi S (2013) Role of maspin in cancer. Clin Trans Med 2: 8. https://doi.org/10.1186/2001-1326$2-8$

Bu XM, Zhao CH, Dai XW (2008) Aberrant expression of Wnt antagonist SFRP1 in pancreatic cancer. Chin Med J (Engl) 121: 952-955. PMID: 18706212

Bu XM, Zhao CH, Zhang N, Gao F, Lin S, Dai XW (2008a) Hypermethylation and aberrant expression of secreted frizzled-related protein genes in pancreatic cancer. World J Gastroenterol 14: 3421-3424. https:// doi.org/10.3748/wjg.14.3421

Cho KR, Shih IeM (2009) Ovarian cancer. Annu Rev Pathol 4: 287-313. https://doi.org/10.1146/annurev.pathol.4.110807.092246

Cowling VH, D’Cruz CM, Chodosh LA, Cole MD (2007) c-Myc transforms human mammary epithelial cells through repression of the Wnt inhibitors DKK1 and SFRP1. Mol Cell Biol 27: 5135-5146. https://doi.org/10.1128/MCB.02282-06

Dees C, Schlottmann I, Funke R, Distler A, Palumbo-Zerr K, Zerr P, Lin NY, Beyer C, Distler O, Schett G, Distler JH (2014) The Wnt antagonists DKK1 and SFRP1 are downregulated by promoter hypermethylation in systemic sclerosis. Ann Rheum Dis 73: 1232-1239. https://doi.org/10.1136/annrheumdis-2012-203194

Esteller M (2007) Cancer epigenomics: DNA methylomes and histone-modification maps. Nat Rev Genet 8: 286-298. https://doi. org/10.1038/nrg2005

Esteller M (2008) Epigenetics in Cancer. N Engl J Med 358: 1148-1159. http://doi.org/10.1056/NEJMra072067

Holly VL, Widen SA, Famulski JK, Waskiewicz AJ (2014) Sfrp1a and Sfrp5 function as positive regulators of Wnt and BMP signaling during early retinal development. Dev Biol 388: 192-204. https:// doi.org/10.1016/j.ydbio.2014.01.012

Jerónimo C, Usadel H, Henrique R, Oliveira J, Lopes C, Nelson WG, Sidransky D (2001) Quantitation of GSTP1 methylation in non-neoplastic prostatic tissue and organ-confined prostate adenocarcinoma. J Natl Cancer Inst 93: 1747-1752. https://doi.org/10.1093/ jnci/93.22.1747

Kardum V, Karin V, Glibo M, Skrtic A, Martic TN, Ibisevic N, Skenderi F, Vranic S, Serman L (2017) Methylation-associated silencing of SFRP1 gene in high-grade serous ovarian carcinomas. Ann Diagn Pathol 31: 45-49. https://doi.org/10.1016/j.anndiagpath.2017.07.002

Katoh Y, Katoh M (2006) WNT antagonist, SFRP1, is Hedgehog signaling target. Int J Mol Med 17: 171. https://doi.org/10.3892/ ijmm.17.1.171

Kim SH, Kaschula CH, Priedigkeit N, Lee AV, Singh SV (2016) Forkhead box Q1 is a novel target of breast cancer stem cell inhibition by diallyl trisulfide. J Biol Chem 291: 13495-13508. https://doi. org/10.1074/jbc.M116.715219

Kurman RJ, Shih IeM (2010) The origin and pathogenesis of epithelial ovarian cancer: a proposed unifying theory. Am J Surg Pathol 34: 433-443. https://doi.org/10.1097/PAS.0b013e3181cf3d79

Kurman RJ, Shih IeM (2011) Molecular pathogenesis and extraovarian origin of epithelial ovarian cancer - shifting the paradigm. Human Pathol 42: 918-931. https://doi.org/10.1016/j.humpath.2011.03.003
Lee WH, Morton RA, Epstein JI, Brooks JD, Campbell PA, Bova GS, Hsieh WS, Isaacs WB, Nelson WG (1994) Cytidine methylation of regulatory sequences near the pi-class glutathione $\mathrm{S}$-transferase gene accompanies human prostatic carcinogenesis. Proc Natl Acad Sci U S A 91: 11733-11737. https://doi.org/10.1073/pnas.91.24.11733

Lheureux S, Gourley C, Vergote I, Oza AM (2019) Epithelial ovarian cancer. Lancet 393: 1240-1253. https://doi.org/10.1016/S01406736(18)32552-2

Morgan RJ Jr, Alvarez RD, Armstrong DK, Boston B, Burger RA, Chen LM, Copeland L, Crispens MA, Gershenson D, Gray HJ, Grigsby PW, Hakam A, Havrilesky LJ, Johnston C, Lele S, Matulonis UA, O’Malley DM, Penson RT, Remmenga SW, Sabbatini P, Schilder RJ, Schink JC, Teng N, Werner TL; National Comprehensive Cancer Network (2011) Epithelial Ovarian Cancer. J Natl Compr Canc Netw 9: 82-113. https://doi.org/10.6004/jnccn.2011.0008

Partl JZ, Fabijanovic D, Skrtic A, Vranic S, Martic TN, Serman L (2014) Immunohistochemical expression of SFRP1 and SFRP3 proteins in normal and malignant reproductive tissues of rats and humans. Appl Immunohistochem Mol Morphol 22: 681-687. https://doi. org/10.1097/PAI.0000000000000019

Revet I, Huizenga G, Koster J, Volckmann R, van Sluis P, Versteeg R, Geerts D (2010) MSX1 induces the Wnt pathway antagonist genes DKK1, DKK2, DKK3, and SFRP1 in neuroblastoma cells, but does not block Wnt3 and Wnt5A signalling to DVL3. Cancer Lett 289: 195-207. https://doi.org/10.1016/j.canlet.2009.08.019

Satoh W, Matsuyama M, Takemura H, Aizawa S, Shimono A (2010) Sfrp1, Sfrp2, and Sfrp5 regulate the Wnt/beta-catenin and the planar cell polarity pathways during early trunk formation in mouse. Genesis 46: 92-103. https://doi.org/10.1002/dvg.20369

Sugiyama Y, Shelley EJ, Wen L, Stump RJ, Shimono A, Lovicu FJ, McAvoy JW (2013) Sfrp1 and Sfrp2 are not involved in Wnt/ß-catenin signal silencing during lens induction but are required for maintenance of Wnt/ $\beta$-catenin signaling in lens epithelial cells. Dev Biol 384: 181-193. https://doi.org/10.1016/j.ydbio.2013.10.008

Stojadinovic O, Brem H, Vouthounis C, Lee B, Fallon J, Stallcup M, Merchant A, Galiano RD, Tomic-Canic M (2005) Molecular pathogenesis of chronic wounds: the role of beta-catenin and c-myc in the inhibition of epithelialization and wound healing. Am J Pathol 167: 59-69. https://doi.org/10.1016/s0002-9440(10)62953-7

Tellez CS, Shen L, Estécio MR, Jelinek J, Gershenwald JE, Issa JP (2009) CpG island methylation profiling in human melanoma cell lines. Melanoma Res 19: 146-155. https://doi.org/10.1097/ cmr.0b013e32832b274e

Tetsu O, McCormick F (1999) Beta-Catenin regulates expression of cyclin D1 in colon carcinoma cells. Nature 398: 422-426. https://doi. org/10.1038/18884

Wang Z, Li R, He Y, Huang S (2018) Effects of secreted frizzled-related protein 1 on proliferation, migration, invasion, and apoptosis of colorectal cancer cells. Cancer Cell Int 18: 48. https://doi. org/10.1186/s12935-018-0543-x

Yamaguchi T, Mukai H, Yamashita S, Fujii S, Ushijima T (2015) Comprehensive DNA methylation and extensive mutation analyses of HER2-positive breast cancer. Oncology 88: 377-384. https://doi. org/10.1159/000369904 88:377-384 